\title{
Applying Biological Paradigms to Emerge Behaviour in RoboCup Rescue Team
}

\author{
Francisco Reinaldo ${ }^{1,2}$, Joao Certo ${ }^{1}$, Nuno Cordeiro ${ }^{1}$, Luis P. Reis ${ }^{1}$, Rui Camacho ${ }^{1}$, \\ and Nuno $\mathrm{Lau}^{3}$ \\ ${ }^{1}$ LIACC-NIAD\&R, Faculty of Engineering, University of Porto, \\ R. Dr. Roberto Frias, 4200-465 Porto, Portugal \\ reinaldo.opus@gmail.com, nuno_cordeiro@hotmail.com, \\ \{joao.certo, lpreis, rcamacho\} @fe.up.pt \\ ${ }^{2}$ GIC, Dept. of Computer Science, UnilesteMG, \\ Av. Pres. Tancredo Neves, $\mathrm{n}^{\circ} 3500,35170-056$ Cel. Fabriciano - MG, Brasil \\ ${ }^{3}$ IEETA/DET, University of Aveiro, 3810-193 Aveiro, Portugal \\ lau@det.ua.pt
}

\begin{abstract}
This paper presents a hybrid behaviour process for performing collaborative tasks and coordination capabilities in a rescue team. RoboCup Rescue simulator and its associated international competition are used as the testbed for our proposal. Unlike other published work in this field one of our main concerns is having good results on RoboCup Rescue championships by emerging behaviour in agents using a biological paradigm. The benefit comes from the hierarchic and parallel organisation of the mammalian brain. In our behaviour process, Artificial Neural Networks are used in order to make agents capable of learning information from the environment. This allows agents to improve several algorithms like their Path Finding Algorithm to find the shortest path between two points. Also, we aim to filter the most important messages that arise from the environment, to make the right choice on the best path planning among many alternatives, in a short time. A policy action was implemented using Kohonen's network, Dijkstra's and D* algorithm. This policy has achieved good results in our tests, getting our team classified for RoboCup Rescue Simulation League 2005.
\end{abstract}

\section{Introduction}

Search and rescue of victims in large-scale disaster are serious and very difficult tasks presenting several challenges from a scientific point of view. Unprepared cities can suffer tremendous consequences in a natural catastrophe as was reported in Kobe's earthquake [8]. Every city needs an emergency plan, to reduce the loss of human life in a natural disaster. In recent years, staggering technological breakthroughs brought some science fiction dreams closer to us. The innovations in robotics and artificial intelligence have opened doors and allowed for a complete new use of rescue agents and emergency plans.

RoboCup Rescue international project was started in 1999 to solve disaster and rescue problems by integration of disaster information, prediction, planning and 
training for rescue actions. Built upon the success of RoboCup Soccer project, it aims to offer a comprehensive urban disaster simulator, forums of technical discussions and competitive evaluation for researchers and practitioners.

This paper presents our team and its decision system to support planning/control of tasks. The system uses learning modules and path finding algorithms. The use of Kohonen's network [10], Feedforward network, Dijkstra's algorithm [4] and the D* (Dynamic $A^{*}$ ) algorithm [14] are discussed, to provide an understanding of how they are applied in agents. Consequently, collaborative actions are emerged and optimal strategies are achieved with high performance for path-finding and other related tasks.

The rest of the paper is as follow. Section 2 presents an overview of RoboCup Rescue Simulation System. Section 3 shows rescue teams and rescue behaviours. Section 4 provides an overview of Artificial Neural Networks, showing the similarity between rescue team and human brain to validate the choice of Kohonen's network for emergent reasoning and Feedforward for producing simple behaviours. Section 5 introduces our team, giving contributions in: functionalities, structure and layer learning building. In Section 6 we present some results and conclude the paper.

\section{RoboCup Rescue Simulation System}

RoboCup Rescue Simulation League is a rescue project that simulates an urban disaster, heterogeneous team agents and human behaviours [24]. Proposed by Kitano et al. [7], it looks after autonomous agents, complex high-level plans and adjustment of heterogeneous behaviours to save citizens in disasters and to preserve the town (Figure 1). Every year a competition is organized where researchers meet, compare approaches and exchange ideas.

Some emergent methodologies and/or behaviour architectures try to offer suitable solutions for providing emergency decision support. But an unconcern with the requirements of disaster simulation or the topologic structure of the system can suggest an arduous policy of solution elaboration process in the simulation phase.

Challenges and requirements are the guidelines of the rescue simulation system and competition. The challenges are: limited time for rescuing injured citizens; simulation of rescue behaviours, centre agents and team agents; best route planning for moving vehicles allowing detours around blocked ways; distribution of water to extinguish burning buildings; and others. In order to solve this, it is necessary to develop useful reasoning/reactive modules to deliberate/perform secure actions. These modules offer strategies for saving lives and preserving buildings. In addition, Takahashi [24] has presented and categorized, according to their usage, three main classes of requirements in a disaster simulation:

- Before disasters: Prevention plans for disasters and how to supply provisions to refuges. This phase is considered in some way as the agents may have a set of adjustable parameters according to the map of the City in which they act.

- During disasters: Rescue operations at the field are done to save lives, prevent the destruction of households and so on. These operations are estimated to last for the 72 hours immediately after the disaster.

- After disasters: Disasters in urban cities may have effects for a long time. 


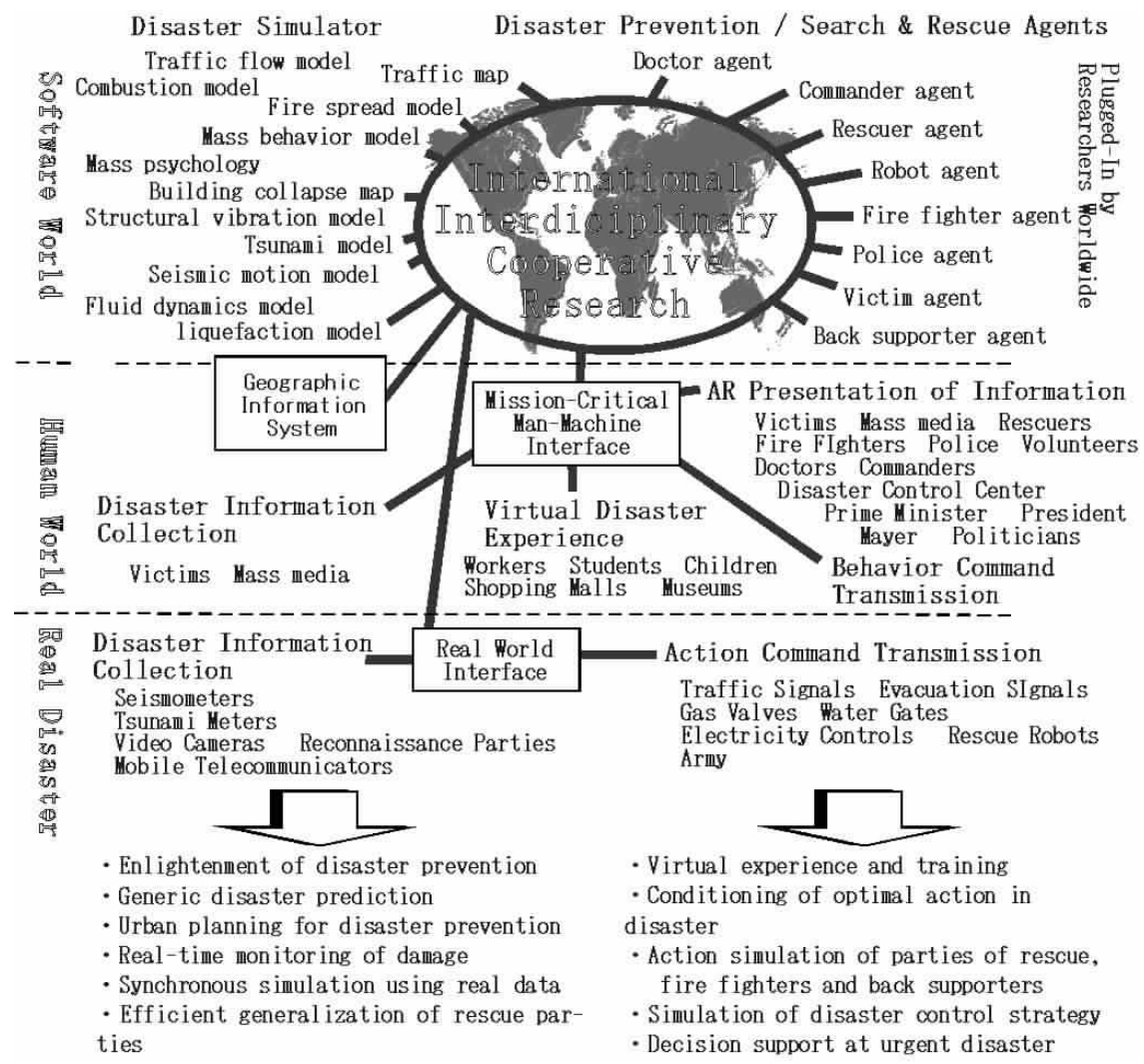

Fig. 1. Concept of the RoboCup Rescue simulation project, extracted from [23]

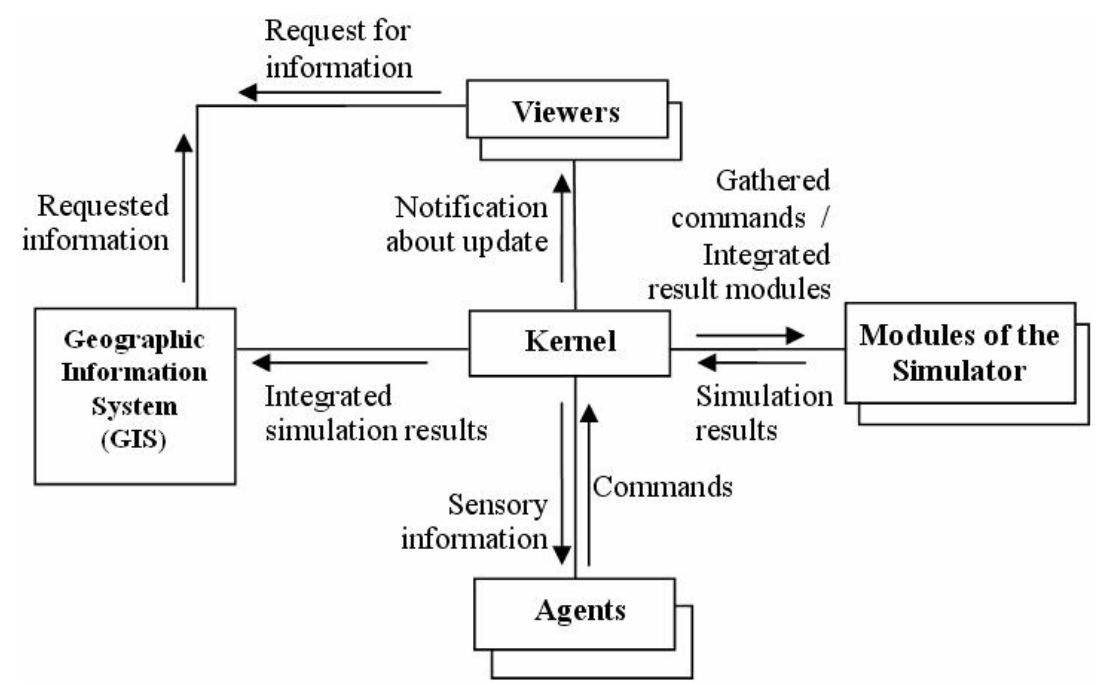

Fig. 2. RoboCup Rescue Simulation System 
The RoboCup Rescue Simulator is a framework that is divided by modules that can run in several computers. These modules are independent to run particular characteristics of the world, as can be seen in figure 2 . The modules also represent simulation domains as earthquake, buildings on fire or collapsing, spreading of fire to neighbouring buildings, blocked ways and health status of citizens.

The main components that comprise the simulator are [3]:

- Geographic Information System (GIS): the GIS module holds the state of the simulated world;

- Kernel: this module is connected to all the other modules. The kernel updates the objects in the GIS and sends the world update for every connected module.

- Sub-simulators: Fire-simulator, Collapse-simulator, Traffic-simulator etc. are modules connected in the kernel, each one simulating a particular disaster feature - fire, collapses, traffic, etc.

- Agents: agent modules are connected to the kernel and represent intelligent entities in the real word, such as civilians, polices agents, fire agents etc.

- Viewers: their task is to get the state of the world, communicating with the kernel module, and graphically displaying it, allowing the user to easily follow the simulation process.

The simulator presents a world model with fire brigades, ambulances, police cars, civilians, roads, buildings and fire, as can be seen on figure 3 .

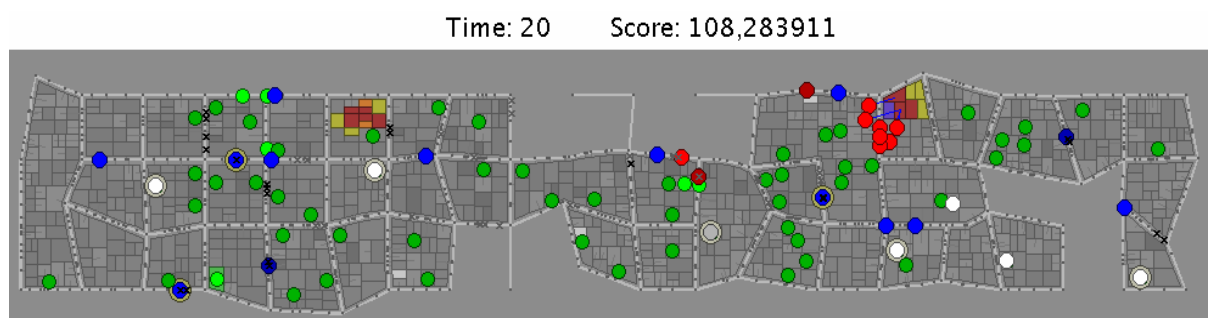

Fig. 3. Rescue Scenario

The RoboCup Rescue domain [2,3] includes approximately 40 agents in six different types to preserve the town and citizens' life: Fire Brigade agents, Police Force agents, Ambulance agents and three different Control Centres. Centre agents coordinate platoons with collaborative tasks, which are based on information from the external world, received by mobile agents. These centre agents send/receive several messages of world state to/from its mobile agents. The updated messages are sent to the rescue agents. Platoons have the autonomy to learn and to decide on the best course of action on unexpected states. Police agents have the responsibility to clear important blocked ways for extreme traffic and to report to base the detection of a citizen crying for help. Ambulance agents search for buried citizens, rescuing and transporting them to refuges. Fire Brigade agents extinguish buildings on fire. Each centre is responsible for its team. All tasks performed by mobile agents are centred on saving lives. 


\section{Related Work}

RoboCup Rescue intends to promote research and development to this socially significant domain at various levels, involving multi-agent team work coordination, development of physical robotic agents for search and rescue, development of information infrastructures, personal digital assistants, and standard rescue simulators [13]. The promotion of an annual rescue challenge becomes a way to get together some different teams for searching the best planned actions to minimize the damage and to test them in a virtual city. The methodical laying of practical strategy and tactics is the key to victory.

There are several teams developing agents, with advanced learning and coordination capabilities, for RoboCup Rescue Simulation. Paquet et al. [15] presented a very good survey concerning the use of coordination methodologies in RoboCup Rescue. Several other teams present very interesting work on applying coordination methodologies to Rescue $[1,5,9,15]$.

DAMAS Rescue team [15] concentrates its work on improving the agent's ability to extinguish buildings on fire. The ability consists in classifying the best fire to extinguish by perception learning method. Agents are using a global view and a specific view decision making process to choose the best fire zone to attack. Global view focuses on the various burning areas. Specific view makes use of more detailed information to choose which specific building to extinguish in the chosen fire zone. The correlation between utility, and expected reward, can be seen as an estimate of the capability to extinguish a given fire. Using perception techniques, the aim is to learn how to coordinate agents, to extinguish the most important fires in a given fire zone. In the development of the agents' plan, DAMAS Rescue team used Jack Intelligent Agent programming language [6], decision tree algorithms and reinforcement learning. During the simulation, the agents use the tree, created offline, to decide the best fire zone and, inside the fire zone, the best building to extinguish. This has the effect of reducing the state space of the reinforcement learning algorithm and, thus, facilitating the learning process. Other interesting point is that fireman doesn't change target areas without a good reason to do so.

ResQ Freiburg [9] deals with a sequence of planning methods. The objective is to build hierarchic commands that mean hierarchic behaviours. The goal of limiting damage to people and buildings is achieved by successfully coordinating teammates by deliberative high-level decisions of the centre agents. The decision about the execution of actions is decomposed in a reactive level by the platoon agents and a deliberative level by the centre agents. Agents have prediction methods instead of classical planning research. This prediction method is an evaluation function for possible targets with respect to the current state of the environment. This function is extended to use hierarchical reinforcement learning. Centre agents decide task execution in the long term by allocating groups of platoon agents to particular tasks. Their decision making is based on a module for state prediction and abstraction, which generates the input for a novel multi-agent planner.

Caspian [5] uses a three phase model approach to develop their agents: World Model, Dynamic Path Finding and Decision Making. The first phase is the development of a World Model. The importance of this phase is crucial as the better the model is, and better the update of that model, the better the decision will be. 
The model for the world is the best for the available sensory information, as every agent knows everything that is sensed by other agents. As the number of messages each agent can listen is limited, this is achieved by centre agents who collect every humanoid agent messages, and other centre agent messages, and send a new one with all the new (based on timestamp) information. Decision Making is only done in humanoid Agents, each of them as a priority assigning algorithm witch chooses the most important (homogeneous) job. Each Police Force agent is assigned a zone to work on. Ambulance Teams always work collectively when rescuing. Their task and coordination is handled by a leader. If there are no civilians to rescue in the world model, each ambulance agent is assigned a zone to search. Fire Brigade behaviour is similar to the one on Ambulance Teams, with the exception that, some times, they get separated into two or more groups.

SOS team [20] develops a general-purpose rescue agent for implementing highlevel strategies and learning algorithms. The SOS agent has a state-based architecture with an explicit state-selection and state switching policy. It uses two path-finding modules that are $\mathrm{D}^{*}$ and Dijkstra's algorithm. The search for fire sources and buried civilians is done by clustering the search function space into regions of equal size. Using the idea of Kohonen's network, a Voronoi diagram is constructed from those clusters. Agents are assigned regions extracted from the diagram. Tasks are prioritized according to their importance. The Fire Brigade agents use a two level priority scheme for increasing the locality of successive tasks, and reducing the amount of time wasted on move actions. After defining a Fiery Region as a connected group of burning buildings, firstly the agents are assigned to a region and secondly to a building on fire. A method of Reinforcement Learning based on Q-Learning is used by Fire Brigade agents, which suffer awards or penalties according to their efficiency in performing tasks. Police agents perform actions by use of centralized and decentralized decision-making rules. After the simulation is started, the Police Office assigns some Police Forces to clear the blocked ways that are around a discovered fire focus. These routes will be used to facilitate the free movement of agents around these critical spots. When no jobs are received from the Police Office, agents scout for fire and buried civilians. In order to decide the best ways to clean, SOS uses a concept of cell temperature that reflects the importance for the overall performance of the team: the higher the temperature the more important and obstructed is that piece of terrain, therefore the agents try to lower the temperature of the hottest cell to an acceptable level. The ambulance teams use an algorithm to estimate the remaining lifetime of injured civilians. This estimation is achieved through the use of a 3-layer BackPropagation Neural Network. In this case, only injured citizens are rescued.

\section{Artificial Neural Network and Biologic Plausibility}

Some algorithmic methods do not give a suitable solution to multiagent problems because the agents' actions are largely intricate. The problem must be treated by dividing the problematic phases in specialized modules in order to reduce their complexity. So we turn to Nature, studying Brainstem and Forebrain, to demonstrate why we have used Kohonen's network in centre agents, as can be seen in figure 4. 
An Artificial Neural Network (ANN) is a data processing paradigm that is inspired by biological neural networks of the brain. An ANN has the ability to learn by examples which gives it a lot of power and flexibility. It is composed of several interconnected neurons that perform excitatory and inhibitory connections to adjust their synaptic weights between neurons to solve a problem and to produce the correct output. The massive parallelism of neurons in an ANN structure can offer data compression, because information can be stored in the weights of the connections. Depending on the network type and its topology, an ANN has the capacity to obtain meaning from complex or imprecise inputs and adjust them to any situations or even to model a complex decision system.

Inside mammalian brain, we can find the Brainstem, that is able to create a far simpler sensory world, and Forebrain, that is thought to coordinate advanced cognitive functions, such as thinking, planning and language [11]. Brainstem is divided in Hindbrain and Midbrain. Hindbrain and Midbrain are, essentially, extensions of the Spinal Cord that receive signals from the environment as well. Hindbrain coordinates and supports movements of walking, and Midbrain orients the body to stimuli. This orientation to stimuli requires both sensory input and motor output. Forebrain is divided in Neocortex, Basal Ganglia, Limbic system and Thalamus. All sensory information reaches the Forebrain through the Thalamus in Diencephalon. The Neocortex creates a perceptual world and regulates the mental activities such as perception and complex planning. The Limbic Cortex is thought to play a role in controlling motivational states, and regulating emotions and behaviours that require memory. The Basal Ganglia and Cerebellum modify movements through inhibitory output and excitatory output, respectively, because the motor cortex sends information to both, and both structures send information right back to the cortex via the Thalamus.

In rescue simulation, the agent's orientation is in response to the world state. Teammates use their sensory input to design a far and simpler sensory world. Massive amounts of data are received from the environment and processed to produce oriented behaviours by rescue agents. Data input is sent to centre agents that will produce, actualize and share a perceptual world. In order to produce it, the sensory systems share some sort of common map of the external world. Moreover, centre agents deliberate cognitive functions to coordinate teammates by overlapping their behaviours. The cognitive functions are responsible for the emergence of reasoning, planning, and memorizing/updating messages to soften the communication. These characteristics of deliberation depend of sensory input of teammates. Each register in the world state is associated with a time stamp, so that the most recent information is always the one recorded. Success and punishment of an agent is regulated by activation/inhibition between the units using their connections, as well as processes for modifying the connections. In the whole rescue simulation system, success and punishment allow for smooth and coordinated movement.

This analogy between mammalian brain and rescue simulation demonstrates that the whole rescue simulation system can be manipulated as a biologic system. We can see in figure 4 that the two systems have a similar production of behaviours. This shows that Kohonen's network can be used as a Neocortex in the centre agents because it reaches complex cooperative deductions with learning ability. In this topology, Kohonen's network allows the creation of a self-organized map based on 
input data. This ANN has a massive parallelism in the manner that the neurons are interconnected. Our rescue architecture permits overlapping behaviours in teammates as a hierarchy. These qualities make it a motivating addition to agents as they can progressively adjust themselves to provide a more challenging experience for the FC Portugal Rescue Team simulation.

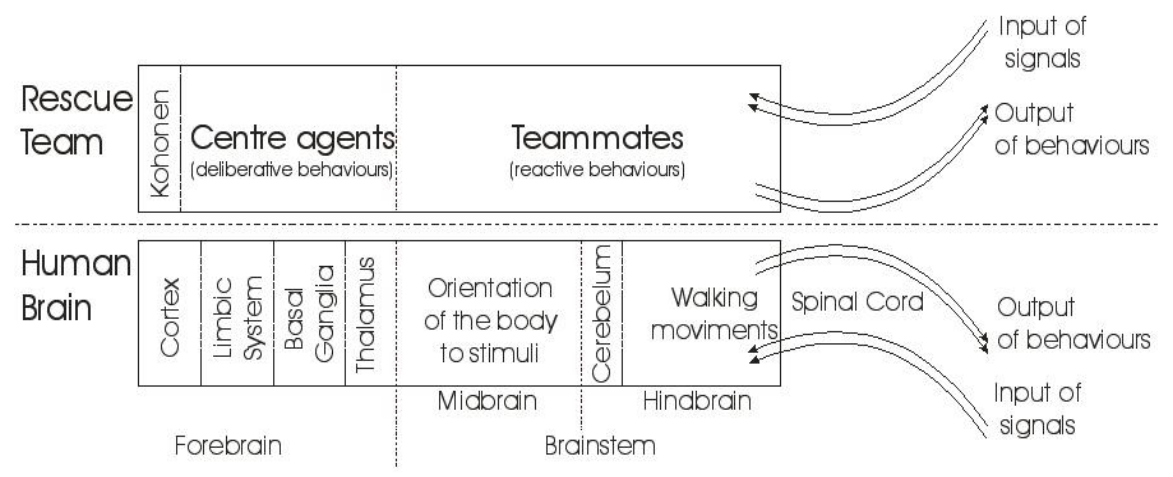

Fig. 4. An analogy between Rescue Team and Human Brain

\section{FC Portugal Rescue Team}

The search and rescue of citizens, in difficult conditions, on a hostile environment, is a very serious social issue, involving a large number of heterogeneous agents, working together as a team. FC Portugal Rescue Team has elaborated its own learning process to improve the exploration of the world by rescue agents. Agents have the ability to acquire a variety of symbols from the environment and manipulate them in order to emerge knowledge. Using a connectionist approach and path finding algorithms, the agents learn about the surrounding environment and consequently decide on new actions to perform.

\subsection{Agent Learning Capabilities}

The learning process of decision making is composed of modules. Those are disposed in a hierarchic and hybrid structure. In accordance with Reinaldo [16], the knowledge is hierarchical from simple to complex. Simple knowledge types used in the rescue domain include the position of burning buildings, blocked roads and buildings with buried people. Complex knowledge may include team formation and communication methods.

This disposition of modules reaches fast negotiation and coordination of behaviours to centre agents and reactivity to team agents. Cooperative behaviours are emerged in deliberative level and can offer a class of predefined behaviours aspects. Predefined behaviours can be formulation of routes, anticipation and modification of plans accordingly to the ability of sharing an inner world mental map. Centre agents with cognitive capabilities can perform better tasks than reactive behaviours in this domain. 
An ANN is robust enough to model a complex collaborative decision, by searching new ways to solve an unknown but similar problem. This quality is devoted to deal with large quantities of data with high performance. Experimental tests involving Kohonen's networks make it clear that the knowledge improves performance and decreases communication among centre and team agents. On the other hand, in a dynamic environment with unknown states and priority of actions, we are using Feedforward. In the routing module, the information provided to an agent includes the positions and neighbours of each building and road in the environment. The combination of $\mathrm{D}^{*}$ path-finding algorithm and Dijkstra's algorithm provides us a hybrid module for finding the best path from origin to destination. In this module there is a limit on the depth of the search tree, for safety.

Dijkstra's algorithm is one of the most efficient algorithms to calculate the minimum cost of the path between vertexes. This algorithm chooses a vertex as root of search and calculates the minimum cost of this vertex for all sequent vertices. It has an acceptable performance but does not offer guaranties of the exact solution if there are negative arcs. This algorithm stipulates an initial approximation to minimum cost and incrementally adjusts this value.

We are using $D^{*}$ algorithm because it plans optimal traverses by repairing paths to the agent's state as new information is discovered in dynamic environments. In accordance with Stentz [22], the algorithm computes an initial path from the goal state to start state and then efficiently modifies this path during the traverse. Stentz had shown, in his research, that this algorithm produces an optimal traverse, meaning that an optimal path to the goal is followed at every state in the traverse, assuming all known information at each step is correct.

\subsection{Functionality and Coordination Methodologies}

We are interested in the research of new methodologies that promote the development of effective, efficient and practical solutions for fast and stable agents. In order to work with lower-level rescue behaviour the details are important, in order to feed the learning methods and instantaneous decisions modules. In addition, higher-level rescue behaviour is responsible for emerging actions by use of coordination modules.

First we are mainly interested in researching new coordination and learning methodologies. In order to abstract from lower-level rescue simulation details, and be able to run efficiently most of our planned algorithms, we need a more efficient language. Thus, we moved to $\mathrm{C} / \mathrm{C}++$ code using, as a reference, Michael Bowling Agent Development Kit [2] and SOS 2003 source code [21]. However since most of our other RoboCup teams $[12,19]$ are implemented using $\mathrm{C}++$, it is a lot easier to adapt our code to a Rescue team implemented also in C++.

Our agents' low-level strategy is mainly the following: at the beginning of the search and rescue operation, Police agents try to free strategic routes, in order to enable ambulance agents and fire brigade agents to move freely between important locations. These points include not only fire spots and civilian refuges, but also map strategic crossings. Ambulance strategy is fairly simple and is based on taking close, severely injured, civilian agents to refuges, following known free paths. The $\mathrm{D}^{*}$ 
algorithm is used in order to find the fastest free known paths for ambulance navigation in the map. Fire brigades try to combat the fire using pre-defined collective plans for: attacking directly a fire, minimizing fire spread, or containing the fire. Fire combat strategy is more elaborate and is based on defining attack perimeters for known fires. If fire is contained, fire brigades are used to search for buried civilians, in order to maximize team global scoring. Centre agents are responsible for message routing and global tactical reasoning for each type of agent.

Our main focus is a high-level methodology that uses complex behaviours, in order to emerge cooperative actions in a coordinated fashion. These coordination methodologies include:

- Concept of Global Situation. A situation is a high-level analysis of the search field that must be simple to perform by all agents, resulting in common global knowledge for all [20], like avoiding fire spreading, attacking fire and other.

- Situation Based Strategic Positioning. This coordination mechanism [12, 17, 20]enables a team of agents to move in a coordinated way in a spatial domain, based on common a-priori tactical knowledge and simple environment knowledge [20].

- Definition of a Team Strategy for a Competition. This strategy is composed by tactics with activation rules. Tactics include several high-level parameters like group mentality, level of risk taken and also the formation to be used in fire attack, sustaining fire in a line, etc. [17, 20]

- COACH UNILANG. Coach Unilang [6][18] was the first high-level standard language introduced in RoboCup for RoboSoccer Team. In a search and rescue scenario, it enables the improvement of team coordination, by allowing a supervisor agent to define the team strategy, before the competition and perform the tactical changes in the team during the execution of a cooperative task by a group of agents. This strategy will be followed by the Centre Agents in order to coordinate the moving agents. Our coach for RoboCup Rescue performs off-line analysis of logfiles showing the team behaviour, and decides the strategy for each rescue operation before the start of the competition.

- ADVCOM. Intelligent Communication is the mechanism to evaluate the lowbandwidth available at these competitions in a communicated world state. The communication is based on agent's deciding the relevance of communicating a given piece of information, by comparing their own world states with a world state constructed using only communication. Based on the differences between these two world states, agents decide which pieces of information to communicate $[12,17]$.

\section{Preliminary Results}

Although there is much work to be done, and our team is only starting to implement some of the proposed ideas, a few changes have already been made to our source code which resulted in some mild improvements as can be seen in the following graph. 


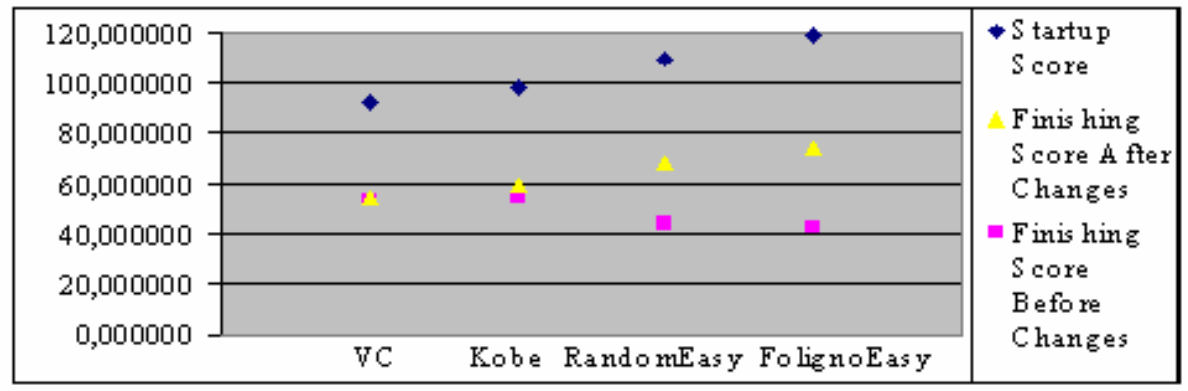

Fig. 5. Score evolution due to improvements in the code

\section{Conclusions and Future Work}

This paper has presented the development of a rescue team using a biological approach, path finding algorithms, and new coordination methodologies aimed at accomplishing a good performance at the rescue championship. We have addressed coordination methodologies with Configurable Flexible Team Strategy and our Situation Based Strategic Positioning to focus on the RoboCup Rescue problem. We are using Kohonen's network and Feedforward network to increase the adaptation in unfamiliar environments by agents and to save the most possible injured citizens. The use of both hierarchic and parallel organisation of behaviours can enable agents to learn information from the environment and subsume behaviours, from centre agents to teammates. Moreover, we have satisfactory results for making the right choice to the best path planning among many alternatives in a short time, using Dijkstra's and $D^{*}$ algorithm. Finally, our code has been fully used by students to promote the advance of freely open source code and speed up of rescue technologies, to minimize the consequences of disasters.

\section{Acknowledgements}

This project is a collaboration between the Univesities of Aveiro and Porto. The authors wish to thank UnilesteMG/BR and FCT/PT for the fellowship granted. This research was supported by FCT under grant \# POSI/EIA/63240/2004. Any opinions, findings and conclusions or recommendations expressed in this material are those of the author(s) and do not necessarily reflect those of the sponsor.

\section{References}

1. Amraii, S. A., Behsaz, B., Gheibi, H., Izadi, M., Janzadeh, H., Molazem, F., Rahimi, A., Ghinani, M. T., and Vosoughpour, H., "S.O.S. 2004: an attempt towards a multi-agent rescue team", Amirkabir Univ. of Tecnology, Tehran, Iran 2004.

2. Bowling, M., "Robocup Rescue: Agent Development Kit", Dept. of Comp. Science, Univ. of Alberta, Alberta, PDF Doc. Avaliabe at http://www-2.cs.cmu.edu/ mhb/research/ rescue/. 
3. d'Agostino, F., Farinelli, A., Grisetti, G., Iocchi, L., and Nardi, D., "Monitoring and information fusion for search and rescue operations in large-scale disasters", in Proceedings of Int. Conf. Information Fusion, AnnaPolis, July 2002, pp. 672-679.

4. Dijkstra, E. W., "A note on two problems in connection with graphs", in Numerische Mathematik 1. Amsterdam, The Netherlands: Mathematisch Centrum, 1959, pp. 269-271.

5. Hamraz, S. H., Feyzabadi, S. S., and Motlagh, A. K., "Caspian RoboCup Rescue Simulation Agent: Team Description", 2005.

6. Howden, N. and Rnnquist, R., "Jack Intelligent Agents - Summary of an agent infrastructure", in Proceedings of 5th Int. Conf. on Autonomous Agents, Montral, Canada, 2001.

7. Kitano, H., Asada, M., Kuniyoshi, Y., Noda, I., Osawa, E., and Matsubara, H., "RoboCup: A Challenge Problem for AI and Robotics", AI Magazine, vol. 18, no. 1, 1997, pp. 73-85.

8. Kitano, H., Hahn, W., Hunter, L., Oka, R., Wah, B., and Yokoi, T., "Grand challenge AI applications", in Proceedings of 13th Int. Conf. on Artificial Intelligence, Chambery, Fr, 1993, pp. 1677-1683.

9. Kleiner, A. and Brenner, M., "ResQ Freiburg: Deliberative Limitation of Damage", Univ. of Freiburg, Freiburg im Breisgau, Germany, PDF Doc. Avaliable at http://www.informatik.uni-freiburg.de/ rescue 2004.

10. Kohonen, T., Self-Organizing Maps, Berlin, Springer, 2001.

11. Kolb, B. and Whishaw, I. Q., An Introduction to Brain and Behavior, 2nd ed., New York, Worth Publishers Inc, 2005.

12. Lau, N. and Reis, L. P., "FC Portugal Homepage", University of Porto, Online at http://www.ieeta.pt/robocup Visited in Oct, 5, 2004.

13. Lau, N., Reis, L. P., and Reinaldo, F., "FC Portugal 2005 Rescue Team Description: Adapting Simulated Soccer Coordination Methodologies to the Search and Rescue Domain", Univ. of Porto, Porto, PDF Doc. Avaliable at http://paginas.fe.up.pt/ lpreis/fcportugal/rescue 2005.

14. Likhachev, M., Ferguson, D., Gordon, G., Stentz, A., and Thrun, S., "Anytime Dynamic A*: An Anytime, Replanning Algorithm", in Proceedings of Int. Conf. on Automated Planning and Scheduling (ICAPS), June 2005, pp. 10.

15. Paquet, S., Bernier, N., and Chaib-draa, B., "DAMAS-Rescue Description Paper", in Proceedings of RoboCup-2004: Robot Soccer World Cup VIII, Springer Verlag, Berlin, 2004, pp. 12.

16. Reinaldo, F. A. F., Projecting a framework and programming a system for development of modular and heterogeneous artificial neural networks, Dept. of Computer Science, Federal Univ. of Santa Catarina, Florianópolis, MSc Thesis, 2003.

17. Reis, L. P. and Lau, N., "FC Portugal Team Description: RoboCup 2000 Simulation League Champion", in Proceedings of RoboCup-2000: Robot Soccer World Cup IV, Berlin, 2001, pp. 29-40.

18. Reis, L. P. and Lau, N., "COACH UNILANG - A Standard Language for Coaching a (Robo)Soccer Team", in Proceedings of RoboCup-2001: Robot Soccer World Cup V, Berlin, 2002, pp. 183-192.

19. Reis, L. P., Lau, N., Costa, P., Nabais, A., Rentes, M., Baixinho, L., Oliveira, E., and Moreira, A. P., "FC Portugal: New Coordination Methodologies in RoboCup Legged League", Univ. of Porto, Porto, Team Description Paper 2005.

20. Reis, L. P., Lau, N., and Oliveira, E., "Situation Based Strategic Positioning for Coordinating a Simulated RoboSoccer Team", in Proceedings of Balancing Reactive and Social Deliberation in MAS, Berlin, 2001, pp. 175-197. 
21. SOS, "Official SOS Home Page", Amirkabir University of Technology, Online at http://ce.aut.ac.ir/ sos/ Visited in Feb 23, 2005.

22. Stentz, A., "The Focussed D* Algorithm for Real-Time Replanning", in Proceedings of Int. Conf. on Artificial Intelligence, Montreal, Canada, Aug. 20-25 1995, pp. 1652-1659.

23. Tadokoro, S., Kitano, H., Takahashi, T., Noda, I., Matsubara, H., Shinjoh, A., Koto, T., Takeuchi, I., Takahashi, H., Matsuno, F., Hatayama, M., Nobe, J., and Shimada, S., "RoboCup-Rescue: An international cooperative research project of robotics and AI for the disaster mitigation problem", in Proceedings of Unmanned Ground Vehicle Technology II, Orlando, FL, USA, 2000, pp. 303-312.

24. Takahashi, T. and Nobe, J., "Evaluation methods for rescue activities by agents and a disaster prevention plan", in Proceedings of 41st SICE, 2002, pp. 858-859. 